\title{
Análise da Agregação de Portadoras do NR-5G usando o ns-3
}

\author{
Daniel R. de Luna, Lucas I. C. Medeiros, Vicente A. de Sousa Jr.
}

\begin{abstract}
Resumo-A quinta geração (5G) da rede de comunicação do 3GPP propõe uma variedade de casos de uso, que vão desde as comunicações móveis em banda larga melhoradas (eMBB), até comunicações ultra confiáveis e de baixíssimo atraso (URLLC), além das comunicações massivas entre máquinas (mMTC). Este trabalho apresenta uma análise de desempenho do uso da agregação de portadoras (CA) no sistema de comunicação 5G NR, utilizando o simulador de eventos discretos ns-3 5G-LENA. Para isso, foram feitas análises com bandas contíguas, considerando taxas oferecidas e distâncias diferentes.
\end{abstract}

Palavras-Chave-5G, NR, ns-3, 5G-LENA, Agregação de Portadoras, Partições de Banda.

Abstract-The fifth generation (5G) of the 3GPP communication network proposes a variety of use cases, from enhanced mobile broadband communications (eMBB), to ultra reliable low delay communications (URLLC), in addition to massive machine type communications (mMTC). This work proposes to develop a performance analysis of the use of carrier aggregation in the 5G NR communication system, using the ns-3 5G-LENA discrete event simulator. For this, analyzes are made with contiguous bands, considering different offered rates and distances.

Keywords-5G, NR, ns-3, 5G-LENA, Carrier Aggregation, Bandwidth Parts.

\section{INTRODUÇÃO}

Conhecida como New Radio (NR), a rede de comunicação do 3GPP (3rd Generation Partnership Project) pretende atender os três casos de uso do $5 \mathrm{G}$ definidos pelo ITU. Essa nova rede, em conjunto com o ecossistema do 3GPP, atenderá desde as comunicações móveis em banda larga melhoradas (enhanced Mobile Broadband - eMBB), até comunicações ultra confiáveis e de baixíssimo atraso (Ultra-Reliable Low-Latency Communication - URLLC), além das comunicações massivas entre máquinas (massive Machine Type Communication - mMTC), comumente conhecida como Internet das coisas (IoT).

As funcionalidades chave do NR incluem redução de sinalização (sinais de referência, sincronização e informações do sistema), suporte para baixo atraso, uso de múltiplas antenas, time-division duplex (TDD) dinâmico e, principalmente, a flexibilidade do uso do espectro. Essa última, estritamente relacionada com a gerência da largura de banda do $5 \mathrm{G}$, se destacando as funcionalidades de agregação

Daniel R. de Luna, Lucas I. C. Medeiros e Vicente A. de Sousa Jr são da Universidade Federal do Rio Grande do Norte, E-mails: \{daniel.luna.088, lucas.medeiros.114, vicente.sousa\}@ufrn.edu.br.

Esse estudo foi financiado em parte pela Coordenação de Aperfeiçoamento de Pessoal de Nível Superior - Brasil (CAPES) - Código de Financiamento 001 .

As simulações fornecidas por esse trabalho foram realizadas com o apoio do Núcleo de Processamento de Alto Desempenho da UFRN (NPAD/UFRN). de portadora (Carrier Aggregation - CA) e a operação em ondas milimétricas, cuja faixa de frequências é chamada de Frequency Range 2 (FR2). Além disso, ainda há a cooperação entre bandas abaixo e acima de $6 \mathrm{GHz}$ e o direcionamento de banda via Bandwidth Part (BWP) para otimizar o gasto energético (reduzindo o processamento em banda base e banda passante) [1].

O caso de uso eMBB, por exemplo, deve suportar uma grande variedade de novos cenários com melhor desempenho, além de uma experiência do usuário aprimorada, quando comparado com as aplicações existentes de comunicações em banda larga. Há cenários em que dependendo das configurações do sistema, as taxas podem atingir picos a $100 \mathrm{Gpbs}$ [2]. Em áreas urbanas densas, por exemplo, é esperado que as taxas sejam em torno de 100 Mbps e 50 Mbps por usuário para downlink e uplink, respectivamente [2]. Para os cenários mais desafiadores, o uso do MIMO (do inglês, Multiple Input Multiple Output) junto da formatação de feixe (do inglês, Beamforming) e das ondas milimétricas (mmWaves) é necessário para atingir taxas tão elevadas. De modo complementar, o uso da CA em bandas contíguas ou não, funciona como mais uma estratégia para dar suporte à essas altas taxas.

Como maneira de avaliar esses cenários sem a necessidade de construí-los fisicamente, o uso de ferramentas de prototipagem via software do sistema de comunicações pode ser utilizado, economizando tempo e custo. Outra vantagem é a possibilidade de, mesmo depois da criação do sistema, testar novas (ou aprimorar antigas) funcionalidades que podem ser incorporadas no padrão, bem como prover soluções para coexistência com sistemas legados. Com tal ferramenta é possível, por exemplo, responder sobre a coexistência de sistemas Wi-Fi, 4G e TV digital com os novos sistemas 5G-NR.

Dentre as ferramentas de prototipagem, destaca-se o network simulator version 3 (ns-3), simulador de eventos discretos open-source, com comunidade ativa, sendo constantemente atualizado com novas funcionalidades [3]. O ns-3 é uma alternativa muito usada ao invés da construção de simuladores próprios, que não são testados exaustivamente pela comunidade externa. Neste trabalho é desenvolvido uma análise de desempenho do uso da CA no sistema de comunicação 5G NR em FR2, utilizando o módulo 5G-LENA para o ns-3. Para isso, são feitas análises com bandas contíguas, considerando diferentes partições de banda, taxas oferecidas e distâncias diferentes.

O artigo está dividido como a seguir: a Seção II apresenta os trabalhos relacionados ao tema deste artigo; a Seção III aborda a modelagem do sistema NR no simulador ns-3, bem como os 
principais parâmetros utilizados nesta investigação; a Seção IV apresenta e discute os resultados, enquanto a Seção V traz as conclusões e comentários finais.

\section{TRABALHOS RELACIONADOS}

Nesta seção são apresentados os principais trabalhos relacionados à este artigo, considerando as duas extensões do ns-3 para os sistemas $5 \mathrm{G}$, desenvolvidas por dois grupos de pesquisa distintos: o ns3-mmwave desenvolvido pela University of Padova e NYU Wireless [4], e o 5G-LENA desenvolvido pelo Centre Tecnològic de Telecomunicacions de Catalunya (CTTC) [5].

\section{A. mmwave-ns3}

Os autores de [6] apresentam a extensão do ns-3 para sistemas $5 \mathrm{G}$ para as ondas milimétricas, o ns3-mmwave. $\mathrm{O}$ trabalho apresenta as etapas do desenvolvimento do simulador, com foco na integração do já consolidado módulo LTE e na flexibilidade das camadas PHY e MAC. As análises presentes mostram o correto funcionamento do módulo em cenários em que há necessidade de controle de congestionamento.

Os autores de [7] e [8] utilizam o ns3-mmwave para avaliar funcionalidades conhecidas do LTE, como a CA, a Dual Connectivity, e o Handover, nos cenários do 3GPP para os sistemas 5G. Além disso, detalhes de implementação também são descritos.

Por fim, os trabalhos [9], [10] propõem uma implementação do modelo de canal MIMO para as faixas das ondas milimétricas utilizadas nos sistemas 3GPP. Em [10], os autores desenvolvem uma análise mais complexa envolvendo variações de modelos de canais e configurações realistas do arranjo de antenas em cenários multi-celulares.

\section{B. $5 G-L E N A$}

Em [11], os autores apresentam a extensão do simulador ns-3, chamado 5G-LENA, para dar suporte ao sistema de comunicação NR, mostrando os resultados de calibração do simulador considerando as diretrizes do 3GPP. Além disso, eles detalham as modificações nas camadas MAC e PHY, quando comparadas com a modelagem do LTE LENA (módulo do LTE do ns-3).

Para uma visão sistêmica do NR, os autores de [12]-[14] apresentam um estudo aprofundado das numerologias e das BWPs, considerando aplicações com tráfegos diferentes. Além das análises que buscam mostrar o desempenho em diversos cenários do $5 \mathrm{G} \mathrm{NR}$, os artigos trazem também detalhes da implementação das funcionalidades do simulador, como o uso da CA e da multiplexação das numerologias. Há também trabalhos mais específicos da camada PHY do NR usando o ns-3, como [15] e [16], que incluem a implementação do canal espacial [15], seguindo a recomendação do 3GPP, e uma nova abstração da camada física para as simulações sistêmicas [16]. Para a camada MAC, os estudos presentes em [17] e [18] trazem o impacto dos escalonadores do $5 \mathrm{G}$ NR na taxa útil e no atraso para tráfegos em downlink e uplink.

Por fim, os artigos [19]-[21] tratam do uso do espectro de frequências não-licenciados no âmbito do $5 \mathrm{G}$. O primeiro é um survey que aponta os desafios e soluções para as tecnologias que deverão utilizar esse espectro, como o LTE-LBT, Wi-Fi e NR-U. Já [20] usa o n-s3 para realizar uma análise da coexistência entre o NR-U e o padrão 802.11ad (WiGig) operando em ondas milimétricas, enquanto que [21] complementa o estudo por meio de mais casos de estudo. É importante destacar que não foram encontrados artigos sobre a técnica de CA, utilizando como ferramenta de prototipagem o ns-3 com 5G-LENA ou ns3-mmwave, após o lançamento do Release 15 do $3 \mathrm{GPP}$.

\section{Modelagem do Sistema NR No NS-3}

Nesta seção é discutido a modelagem do sistema NR no ns-3. Na Seção II foi explicitado quais são os dois grupos de trabalho por trás do desenvolvimento das extensões ns3-mmwave ${ }^{1}$ e 5G-LENA ${ }^{2}$, a parceria $N Y U$ Wireless e University of Padova, e o CTTC, respectivamente. Ambos projetos continuam ativos, sendo disponibilizado o código em seus respectivos repositórios. Em ambas páginas há instruções de instalação e uso dos módulos. Para o 5G-LENA, é necessário ainda solicitar permissão para o uso. Instruções para isso são encontradas em [5].

Os dois módulos continuam sendo atualizados com novas funcionalidades ou correções de antigas. O ns3-mmwave foi o primeiro a ser disponibilizado, já em 2015 (data do primeiro commit). É comum encontrar nas publicações do ns3-mmwave a implementação de soluções próprias para as camadas PHY e MAC, ou seja, soluções que não seguem de maneira exata o padrão definido pelo 3GPP, provavelmente devido ao pioneirismo do módulo. Note que, embora sejam funcionalidades próprias, os resultados indicam que há desempenho compatível com os requisitados pelo $5 \mathrm{G}$ (e prometidos pelo NR). Já a extensão $5 G$-LENA, implementa o módulo chamado $n r$, e foi criada em fevereiro de 2019, depois do Release 15 do 3GPP, seguindo, portanto, as diretrizes do 3GPP. O 5G-LENA inspirado no ns3-mmwave e, atualmente, é o módulo com mais publicações e chances de ser inserido no branch principal do simulador, uma vez que utiliza a versão dev (versão de desenvolvimento).

Sobre as funcionalidades das extensões, é perceptível por meio das publicações, que o ns3-mmwave priorizou implementar as funcionalidades das camadas PHY e MAC personalizadas, baseadas em algumas recomendações do 3GPP e no módulo lte, já bastante consolidado no ns-3. Isso trouxe ao módulo flexibilização de implementação, possibilitando que diferentes sistemas sejam completamente personalizados e parametrizados [4]. Destaque para o suporte de uma grande variedade de canais 3GPP para ondas milimétricas, por exemplo. Enquanto isso, o módulo $n r$ possui as camadas PHY e MAC seguindo as normas do 3GPP, com poucas funcionalidades herdadas do módulo lte do ns-3 [5]. Isso permite que o 5G-LENA possa ser atualizado sem necessidade de alterações no módulo lte, o que torna seu uso mais atrativo. Além disso, possui documentação em consolidação, suporte

\footnotetext{
${ }^{1}$ Disponível em: https://github.com/nyuwireless-unipd/ns3-mmwave

${ }^{2}$ Disponível em: https://gitlab.com/cttc-lena/nr
} 
com comunidade ativa, e atualização de funcionalidades mais frequente.

Diante das vantagens destacadas e de sua aderência com as normas do $3 \mathrm{GPP}$, o simulador ns-3 em conjunto com o $5 G$-LENA foram escolhidos como ferramentas de prototipagem deste trabalho.

Um das principais etapas na modelagem de um sistema de telecomunicações é definir as faixas de frequência e larguras de banda a serem utilizadas. Neste trabalho, cujo objetivo é avaliar o desempenho da técnica de agregação de portadoras em FR2, optou-se por utilizar uma configuração sem uso da CA e outra com o uso da CA. Assim, tentando se aproximar ao máximo de uma aplicação real com a agregação de portadoras na faixa de ondas milimétricas foi escolhida a faixa $\mathbf{n 2 6 1}$, segundo a norma [22]. De modo semelhante, a configuração CA escolhida é a CA_n261, segundo a norma [23], que utiliza a agregação de duas portadoras contíguas na faixa $\mathbf{n 2 6 1}$ do 3GPP. A Tabela I reproduz as faixas de operação das bandas NR em FR2 com base na norma [22], e a Tabela II mostra as configurações utilizadas nas análises deste trabalho. É importante destacar que, em ambos os casos, foi mantido a largura de banda total de interesse e definida para o FR2.

TABELA I

BANDAS DE OPERAÇÃO NR EM FR2

\begin{tabular}{ccc}
\hline $\begin{array}{l}\text { Bandas de } \\
\text { Operação NR }\end{array}$ & Faixa de Operação & Duplexação \\
\hline n257 & $26.5-29.5 \mathrm{GHz}$ & TDD \\
n258 & $24.25-27.5 \mathrm{GHz}$ & TDD \\
n259 & $39.5-43.5 \mathrm{GHz}$ & TDD \\
n260 & $37-40 \mathrm{GHz}$ & TDD \\
n261 & $27.5-28.35 \mathrm{GHz}$ & TDD \\
\hline
\end{tabular}

TABELA II

PARÂMETROS DAS CONFIGURAÇÕES DAS SIMULAÇÕES.

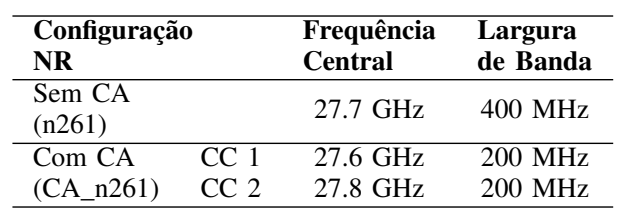

Os parâmetros do cenário de simulação foram configurados de acordo com as diretrizes propostas pelo ITU [24] e 3GPP [25], tendo em vista um cenário indoor-eMBB, pois este é característico das ondas milimétricas. A Tabela III resume os parâmetros gerais utilizados na simulação.

TABELA III

PARÂmetros Gerais de SimulaÇÃo.

\begin{tabular}{lcc}
\hline Parâmetros & Valor & Referência \\
\hline UEs & 1 & - \\
gNBs & 1 & - \\
MIMO & $256(\mathrm{gNB}) \times 32$ (UE) & {$[24]$} \\
Figura de ruído & $7 \mathrm{~dB}(\mathrm{gNB}) \times 10 \mathrm{~dB}(\mathrm{UE})$ & {$[24]$} \\
Altura da antena & $3 \mathrm{~m}(\mathrm{gNB}) \times 1.5 \mathrm{~m}(\mathrm{UE})$ & {$[24]$} \\
Modelo de tráfego & Full Buffer & {$[24]$} \\
Numerologia & 3 & {$[25]$} \\
Beamforming & CellScan (Grid of Beams) & {$[19]$} \\
\hline
\end{tabular}

Em relação aos efeitos de desvanecimento utilizados na simulação, seguiu-se o modelo de canal do 3GPP, especificamente da TR 38.901 [26] para cenários indoor. Nesse documento estão presentes parâmetros e formulações acerca dos cenários $5 \mathrm{G}$ propostos pelo 3GPP, sendo os principais: as equações da perda de percurso com linha de visada (Line of Sight - LOS) e sem linha de visada (Non Line of Sight - NLOS), probabilidade LOS, mobilidade e taxa de atualização do canal. Os dois últimos intrinsecamente relacionados aos efeitos de pequena escala de um canal rádio-móvel. Assim, são propostos três casos de estudo para avaliar a degradação de um sistema NR em downlink para cenários indoor com diferentes intensidades de desvanecimento: 1) apenas perda de percurso; 2) perda de percurso e sombreamento; e 3) perda de percurso, sombreamento e desvanecimento rápido. A Tabela IV traz os principais parâmetros do canal utilizados.

TABELA IV

PARÂMETROS DO CANAL 3GPP [26].

\begin{tabular}{ccc}
\hline Parâmetro & Valor & Descrição \\
\hline Perda de Percurso & InH-Office Mixed & Cenário 3GPP \\
& $\sigma_{S F} 8 d B$ (NLOS) & Descritórios \\
Sombreamento & $\sigma_{S F}=3 d B$ (LOS) & do sombreamento \\
& $d_{\text {cor }}=6 \mathrm{~m}$ (NLOS) & Distância de \\
descorrelação \\
Sombreamento & $d_{C o r}=10 \mathrm{~m}$ (LOS) & do Shadowing \\
& Indoor Mixed office & $\begin{array}{c}\text { Probabilidade de } \\
\text { linha de visada }\end{array}$ \\
LOS probability & Tabela 7.4.2-1 de [26] & Delay e DOA \\
Desvanecimento de & Cluster-based & spreads específicos \\
pequena escala & Tabela 7.5-6 Part-2 [26] & Tempo de coerência \\
Desvanecimento & $100 \mathrm{~ms}$ & do canal \\
\hline
\end{tabular}

Para todos os casos estudados, o cenário avaliado é o mesmo com modificações pontuais para se adequar a avaliação de cada estudo. O deployment avaliado envolve três (3) nós no sistema: um UE, uma gNB e um servidor remoto, que gera o tráfego. A técnica de agregação de portadoras é utilizada como estratégia para melhoria do desempenho do sistema, agregando múltiplas bandas com o objetivo de atingir os requisitos impostos pelo 5G. No ns-3 com o 5G-LENA, o uso da CA está ligado à agregação das partições de banda (BWPs). Nas simulações são criadas duas partições, que são agregadas para avaliar o uso de duas CCs (Component Carriers). São avaliadas situações com e sem a presença da técnica de agregação de portadoras com diferentes taxas oferecida nos três casos de estudo já explanados, com cada caso incrementando a presença de efeitos de degradação no canal de comunicação. São realizadas repetições de cada experimento para garantir um intervalo de confiança de $95 \%$ aceitável (omitido do gráfico para evitar poluição visual). Esse estudo é muito importante para quem trabalha com simulações, pois a modelagem de canal completa é complexa e onerosa em termos de tempo de simulação. Por exemplo, o cálculo da matriz do canal requer recursos de hardware que apenas clusters computacionais podem prover. Além disso, o aumento da complexidade do cenário traz, consequentemente, um aumento na duração das simulações, sendo assim necessário que o projetista esteja atento ao tradeoff entre os principais parâmetros do sistema analisado. Assim, entender qual o impacto de cada modelo 
de canal é importante para avaliar a qualidade da análise de desempenho dos estudos sobre funcionalidades do $5 \mathrm{G}$.

\section{RESUltados}

Nesta seção, os resultados da análise de desempenho para cenários com e sem agregação de portadoras com efeitos de canal diferentes são apresentados e estão condensados nos seus respectivos gráficos. Eles ilustram a degradação do desempenho de um sistema NR, utilizando as ondas milimétrica quando há aumento da distância. Essa avaliação é feita por meio de curvas da taxa útil para taxas oferecidas diferentes, para um cenário indoor (característicos das ondas milimétricas) com ou sem agregação de portadoras. Os principais parâmetros das simulações seguem o estabelecido nas Tabelas III e IV com dados de [25], [26].

Na Fig. 1 são mostrados os resultados da degradação do desempenho pelo aumento da distância entre o UE e a gNB, para taxas oferecidas diferentes, quando considera-se apenas o efeito da perda de percurso. Note que para pequenas distâncias e pequenas taxas oferecidas, não há diferença de desempenho quando comparadas os sistemas com ou sem CA, ou seja, o uso de CA é opcional para essas situações, isso se deve a baixa carga do sistema e a inexistência de escassez de recursos de transmissão. Contudo, ao se oferecer taxas mais elevadas e aumentar a distância entre os dispositivos, uma diferença de desempenho entre as curvas com e sem CA começa a ser notada. Note que embora a banda total do sistema se mantenha, ao usar a CA é possível evitar perdas para as altas taxas oferecidas e grandes distâncias, um dos focos do eMBB no 5G. Importante destacar que cada $\mathrm{CC}$ possui um escalonador próprio, assim quando o CA não é utilizado, a banda total é controlada por um único escalonador, perdendo uma possível diversidade na alocação de recursos. Já com CA, é possível se adaptar a diferentes condições do canal que podem se apresentar nas diferentes CCs. Assim, o escalonador pode alocar os melhores esquemas de codificação e modulação (do inglês, Modulation and Coding Schemes - MCS) para cada uma das CCs.

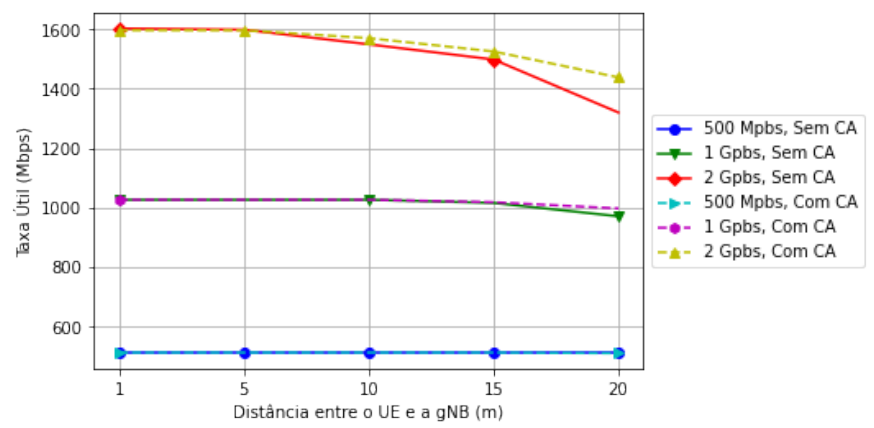

Fig. 1. Taxa útil para cenário $5 \mathrm{G}$ NR sem CA $(1$ x $400 \mathrm{MHz})$ e com CA $(2 \times 200 \mathrm{MHz})$ para o efeitos da perda de percurso.

Já na Na Fig. 2 são mostrados os resultados da degradação do desempenho pelo aumento da distância entre o UE e a gNB, para taxas oferecidas diferentes, quando considera-se os efeito da perda de percurso e sombreamento. A adição do sombreamento ao leque de efeitos de desvanecimento trouxe ao sistema uma degradação notada principalmente para distâncias superiores a 5 , principalmente para configurações sem CA. Contudo, ao chegar na borda de célula, o desempenho, para a taxa oferecida de 2 Gbps, se assemelha com a curva do cenário anterior, em que apenas perda de percurso estava presente. Além disso, como esperado, o uso da CA mantém o desempenho do sistema em patamar melhor do que a configuração sem CA, tendo resultados semelhantes ao desempenho da Fig. 1. Tanto escalonador, quanto a adaptação de enlace da camada MAC são de vital importância para melhor adaptação do MCS em resposta ao canal mais desafiador, indicando que a CA pode ser bem aproveitado, mesmo em situações de canal menos propícias.

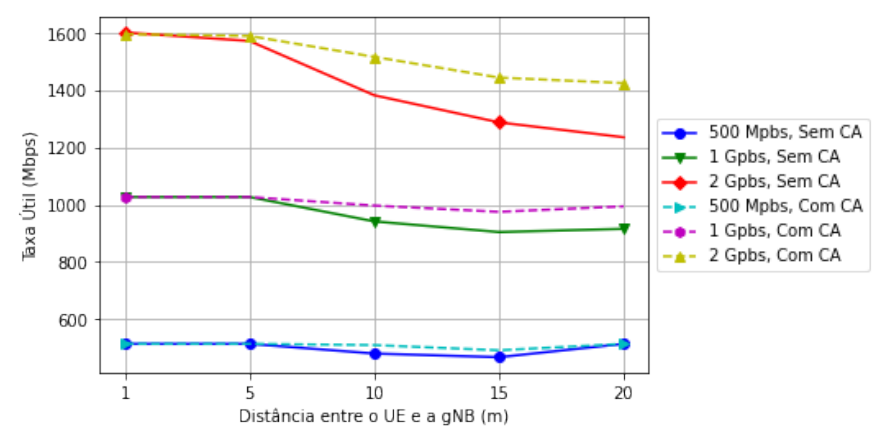

Fig. 2. Taxa útil para cenário $5 \mathrm{G}$ NR sem CA (1 x $400 \mathrm{MHz})$ e com CA $(2 \times 200 \mathrm{MHz})$ para os efeitos de perda de percurso e sombreamento.

Por fim, a Fig. 3 mostra o comportamento da taxa útil considerando o aumento da distância entre a UE e a gNB para cenários com e sem CA na presença de todos os efeitos de canal (perda de percurso, sombreamento e desvanecimento rápido). Claramente, há uma degradação considerável para as curvas de 1 Gbps e 2 Gbps sem CA, enquanto que as curvas com CA embora também afetadas, a degradação ocorreu em um nível menor. As mesmas conclusões relacionados ao escalonador e a adaptação de enlace podem ser tomadas para esse cenário também. Além disso, vale notar que, para todos os cenários analisados, apenas para pequenas distâncias a taxa útil do sistema se igualou a taxa oferecida.

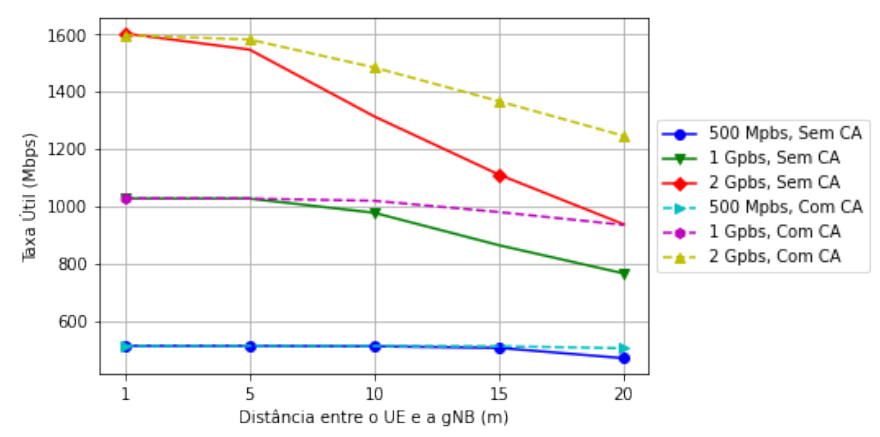

Fig. 3. Taxa útil para cenário $5 \mathrm{G}$ NR sem CA (1 x $400 \mathrm{MHz})$ e com CA $(2 \times 200 \mathrm{MHz})$ para os efeitos de perda de percurso, sombreamento e desvanecimento rápido.

Ao comparar as Figs. 1, 2 e 3, é importante notar que o ganho da CA é maior ao considerar o canal de comunicação completo (perda de percurso, sombreamento e 
desvanecimento de pequena escala). Isso comprova que existe diferença na conclusão quantitativa ao fazer análise de uma funcionalidade de rede para diferentes modelos de canal. Este artigo demonstrou que essa diferença se apresenta mesmo ao analisar funcionalidades que não sejam exclusivamente de camada PHY.

\section{CONClusões}

Este trabalho se propôs a desenvolver uma análise de desempenho do uso da CA no sistema de comunicação 5G NR, utilizando o simulador de eventos discretos ns-3 5G-LENA. Para isso, foram feitas análises com bandas contíguas, considerando diferentes taxas oferecidas, distâncias e efeitos de desvanecimento. Conclusões clássicas também foram encontradas nos sistemas $5 \mathrm{G}$ NR, como a melhoria dos sistemas com o uso da CA e a degradação da taxa útil com a distância. Contudo, vale notar que houve uma estabilização da degradação pela distância com o uso da CA, mantendo a taxa útil em um patamar superior ao encontrado nas configurações sem CA. Esse melhor desempenho reforça o uso da técnica para melhoria do desempenho geral do sistema. O estudo apresentado neste artigo também comprova que existe diferença na conclusão quantitativa ao fazer análise de uma funcionalidade de rede para diferentes modelos de canal, sendo importante, mesmo que oneroso em termos computacionais, a inclusão do canal de comunicação completo. Em trabalhos futuros, é possível explorar as possibilidades de configuração da CA, considerando as formas não-contíguas inter-band e intra-band, este último sendo utilizado em portadoras localizadas tanto FR1 quanto em FR2 ou em ambos. Além disso, é possível avaliar seu uso em cenários densos e heterogêneos para os casos de uso mMTC e URLLC, por exemplo.

\section{REFERÊNCIAS}

[1] X. Lin, A. Grovlen, K. Werner, J. Li, R. Baldemair, J. F. T Cheng, S. Parkvall, D. C. Larsson, H. Koorapaty, M. Frenne, and S. Falahati, "5G New Radio: Unveiling the Essentials of the Next Generation Wireless Access Technology," IEEE Communications Standards Magazine, vol. 3, no. 3, pp. 30-37, 2019.

[2] H. Holma, 5G Technology 3GPP New Radio, 2020.

[3] ns 3. (2021, Abril) Network Simulator version 3. https://www.nsnam.org/.

[4] ns3 mmwave. (2021, Abril) Nyu wireless and university of padova. [Online]. Available: https://wireless.engineering.nyu.edu/e2e-network-simulation/

[5] 5G-LENA. (2021, Abril) Centre tecnològic de telecomunicacions de catalunya. [Online]. Available: https://5g-lena.cttc.es/

[6] M. Mezzavilla, M. Zhang, M. Polese, R. Ford, S. Dutta, S. Rangan, and M. Zorzi, "End-to-end simulation of 5G mmWave networks," IEEE Communications Surveys and Tutorials, vol. 20, no. 3, pp. 2237-2263, 2018.

[7] T. Zugno, M. Polese, and M. Zorzi, "Integration of carrier aggregation and dual connectivity for the ns-3 mmWave module," ACM International Conference Proceeding Series, no. Dc, pp. 45-52, 2018.

[8] M. Polese, M. Mezzavilla, and M. Zorzi, "Performance comparison of dual connectivity and hard handover for LTE-5G tight integration," Proceedings of the 9th EAI International Conference on Simulation Tools and Techniques, SIMUTools 2016, vol. 3, pp. 118-123, 2016.

[9] M. Zhang, M. Polese, M. Mezzavilla, S. Rangan, and M. Zorzi, "Ns-3 implementation of the 3GPP MIMO channel model for frequency spectrum above $6 \mathrm{GHz}$," ACM International Conference Proceeding Series, vol. Part F1283, pp. 71-78, 2017.
[10] M. Rebato, M. Polese, and M. Zorzi, "Multi-Sector and Multi-Panel Performance in 5G mmWave Cellular Networks," 2018 IEEE Global Communications Conference, GLOBECOM 2018 - Proceedings, 2018.

[11] N. Patriciello, S. Lagen, B. Bojovic, and L. Giupponi, "An E2E simulator for 5G NR networks," Simulation Modelling Practice and Theory, vol. 96, 2019.

[12] N. Patriciello, S. Lagen, L. Giupponi, and B. Bojovic, "5G New Radio Numerologies and their Impact on the End-To-End Latency," IEEE International Workshop on Computer Aided Modeling and Design of Communication Links and Networks, CAMAD, vol. 2018-Septe, 2018.

[13] B. Bojovic, S. Lagen, and L. Giupponi, "Implementation and evaluation of frequency division multiplexing of numerologies for $5 \mathrm{G}$ new radio in ns-3," ACM International Conference Proceeding Series, pp. 37-44, 2018.

[14] S. Lagen, B. Bojovic, S. Goyal, L. Giupponi, and J. Mangues-Bafalluy, "Subband Configuration Optimization for Multiplexing of Numerologies in 5G TDD New Radio," IEEE International Symposium on Personal, Indoor and Mobile Radio Communications, PIMRC, vol. 2018-Septe, 2018.

[15] M. Polese, N. Patriciello, S. Lagen, and M. Zorzi, "Implementation of a Spatial Channel Model for ns-3," vol. 3, no. Wns3, 2020.

[16] S. Lagen, K. Wanuga, H. Elkotby, S. Goyal, N. Patriciello, and L. Giupponi, "New Radio Physical Layer Abstraction for System-Level Simulations of 5G Networks," 2020.

[17] N. Patriciello, S. Lagen, L. Giupponi, and B. Bojovic, "An improved MAC layer for the 5G NR ns-3 module," ACM International Conference Proceeding Series, pp. 41-48, 2019.

[18] — " "The impact of NR Scheduling Timings on End-to-End Delay for Uplink Traffic," no. c, 2019.

[19] S. Lagen, L. Giupponi, S. Goyal, N. Patriciello, B. Bojovic, A. Demir and M. Beluri, "New Radio Beam-based Access to Unlicensed Spectrum: Design Challenges and Solutions," IEEE Communications Surveys \& Tutorials, vol. PP, no. c, p. 1, 2018. [Online]. Available: http://arxiv.org/abs/1809.10443

[20] S. Lagen, N. Patriciello, and L. Giupponi, "Cellular and Wi-Fi in Unlicensed Spectrum : Competition leading to Convergence," 2020.

[21] N. Patriciello, S. Lagen, B. Bojovic, and L. Giupponi, "NR-U and IEEE 802.11 technologies coexistence in unlicensed mmWave spectrum: Models and evaluation," IEEE Access, vol. 8, pp. 71 254-71 271, 2020.

[22] 3GPP TS 138 104, "5G; NR; Base Station (BS) radio transmission and reception (Release 15)," 3rd Generation Partnership Project (3GPP), TS 138104 - V15.5.0, vol. 0, pp. 1-219, 2019.

[23] 3GPP TS 38.101-2, "TS 38 101-2 - V16.6.0 - 5G; NR; User Equipment (UE) radio transmissions and reception (3GPP TS 38.101-2 version 16.6.0 Release 16)," $3 G P P$, vol. 0, 2021. [Online]. Available: http://www.etsi.org/standards-search

[24] ITU-R, "Guidelines for evaluation of radio interface technologies for IMT-2020 (Report ITU-R M.2412-0)," vol. 1, p. 144, 2017. [Online]. Available: https://www.itu.int/pub/R-REP-M.2412

[25] ETSI, "5G; Study on Scenarios and Requirements for Next Generation Access Technologies (3GPP TR 38.913 version 14.2.0 Release 14," vol. 0, p. 41, 2017. [Online]. Available: http://www.etsi.org/standards-search

[26] 3GPP TR 38.901, "TR 138901 - V16.1.0 - 5G; Study on channel model for frequencies from 0.5 to $100 \mathrm{GHz}$ (3GPP TR 38.901 version 16.1.0 Release 16)," 3Gpp, vol. 0, 2020. [Online]. Available: http://www.etsi.org/standards-search 